\title{
New Platforms Integrating Ethynyl-grafted Modules for Organogels and Mesomorphic Superstructures
}

\author{
Franck Camerel, Gilles Ulrich and Raymond Ziessel \\ Laboratoire de Chimie, Moléculaire, Associé au CNRS, Université Louis \\ Pasteur, Ecole de Chimie, Polymères, Matériaux de Strasbourg (ECPM), 25, rue \\ Becquerel, 67087 Strasbourg Cedex 02, France \\ ziessel@chimie.u-strasbg.fr

\section{Supporting Information}

\section{General.}

The 300 and $200.1\left({ }^{1} \mathrm{H}\right), 75.47\left({ }^{13} \mathrm{C}\right) \mathrm{MHz}$ NMR spectra were recorded at room temperature with perdeuterated solvents with residual protiated solvent signals providing internal references.

Differential Scanning Calorimetry (DSC) was performed on a Perkin-Elmer DSC-7 instrument. The samples were examined at a scanning rate of $10 \mathrm{~K} \cdot \mathrm{min}^{-1}$ by applying two heating and one cooling cycle. The apparatus was calibrated with indium $\left(156.6{ }^{\circ} \mathrm{C}\right)$ and gallium $\left(29.8{ }^{\circ} \mathrm{C}\right)$. ThermoGravimetric Analyses (TGA) were performed on SDTQ 600 apparatus at scanning rate of $10 \mathrm{~K} \cdot \mathrm{min}^{-1}$ under argon.

Phase behaviour was studied by polarized light optical microscopy (POM) on a Leitz microscope equipped with a Mettler-Toledo FP80 hot-stage and an FP80 central processor.

UV-vis spectra were recorded using a UVIKON 940/941 dual-beam grating spectrophotometer (Kontron Instruments) with a $1 \mathrm{~cm}$ quartz cell. 
$\alpha_{D}$ measurements were performed using a Perkin-Elmer 341 polarimeter on solution at a concentration of $1 \mathrm{~g} / \mathrm{L}$ in dichloromethane $\left(\lambda_{\mathrm{Na}}=589 \mathrm{~nm}\right)$.

FT-IR spectra were recorded using a Perkin-Elmer "spectrum one" spectrometer on the neat liquids or as thin films, prepared with a drop of dichloromethane and evaporated to dryness on $\mathrm{KBr}$ pellets.

2,6 dinitro-4-iodo-toluene ${ }^{[1]}$ and 3,4,5 trialkyloxybenzoic chloride with $\mathrm{C}_{8}, \mathrm{C}_{12}$ and $\mathrm{C}_{16}$ chains ${ }^{[2]}$ were synthesized as previously reported.

\section{Preparation of the Compounds}

\section{2,6-diamino-4-iodo-toluene (1)}

2,6-dinitro-4-iodo-toluene (1g, $3.2 \mathrm{mmol})$ was dispersed in hydriodic acid $(20 \mathrm{~mL}, 57 \%$ in water, unstabilized). The mixture was heated under stirring at $110^{\circ} \mathrm{C}$ for 16 hours. The mixture was poured in ethyl acetate $(100 \mathrm{~mL})$ and was washed with a mixture of satured aqueous solution of $\mathrm{Na}_{2} \mathrm{~S}_{2} \mathrm{O}_{3}$ and $\mathrm{NaHCO}_{3}(50 / 50,100 \mathrm{~mL})$. The organic phase was extracted with aqueous $\mathrm{HCl}(10 \%, 3 \times 100 \mathrm{~mL})$. The resulting aqueous fraction was neutralized with $\mathrm{NaOH}$ saturated solution to reach $\mathrm{pH}=10$ and then extracted with ethyl acetate. After drying the organic phase over $\mathrm{MgSO}_{4}$, removal of the solvent afforded the desired compound as an orange powder $(0.732 \mathrm{~g}, 91 \%) .{ }^{1} \mathrm{H} \mathrm{NMR}\left(200.1 \mathrm{MHz}, \mathrm{CDCl}_{3}\right) \delta 1.91\left(\mathrm{~s}, 3 \mathrm{H}, \mathrm{CH}_{3}\right), 3.55(\mathrm{~s}$, $\left.4 \mathrm{H}, \mathrm{NH}_{2}\right), 6.53(\mathrm{~s}, 2 \mathrm{H}, \mathrm{CH}) .{ }^{13} \mathrm{C}\left\{{ }^{1} \mathrm{H}\right\}$ DEPT NMR (75.47 MHz, $\left.\mathrm{CDCl}_{3}\right) 10.1\left(\mathrm{CH}_{3}\right), 91.2(\mathrm{Cq}-$ I), $106.5(\mathrm{Cq}), 115.2(\mathrm{CH}), 146.4\left(\mathrm{Cq}-\mathrm{NH}_{2}\right)$. UV-vis $\left(\mathrm{CH}_{2} \mathrm{Cl}_{2}, 23{ }^{\circ} \mathrm{C}\right): \lambda_{\max }\left(\varepsilon, \mathrm{M}^{-1} \mathrm{~cm}^{-1}\right)=278$ (19860). IR (KBr) : $3455(v N H), 3377(v N H), 2915(v C H), 2853(v C H), 1613,1572(\delta N H)$, $1487,1421,1314,1254,1191,1157,1102 \mathrm{~cm}^{-1} . \mathrm{MS}(\mathrm{FAB}+, m \mathrm{NBA}): \mathrm{m} / z(\%)=249.1(100)$

\footnotetext{
${ }^{1}$ Arotsky, J. ; Butler, R.; Darby, A. C. J. Chem.. Soc. C 1970, 10, 1480.

${ }^{2}$ Nguyen, H.T., Destrade, C., Malthête J., Adv. Mater., 1997, 9, 375 ; A. El-Ghayoury, Ph.D. Thesis, Université Louis Pasteur, Strasbourg, France, 1999.
} 
$[\mathrm{M}+\mathrm{H}]^{+}$. Anal. Calcd for $\mathrm{C}_{7} \mathrm{H}_{9} \mathrm{IN}_{2}: \mathrm{C}, 33.89 ; \mathrm{H}, 3.66 ; \mathrm{N}, 11.29 ;$ Found $\mathrm{C}, 33.94 ; \mathrm{H}, 3.56 ; \mathrm{N}$, 11.07.

\section{Methyl 3,4,5-(3,7-(S)-dimethyl-6-octenyloxy) benzoate (2)}

(S)-(+)-citronellylbromide (1.00 g, $4.5 \mathrm{mmol})$, methyl 3,4,5-trihydroxybenzoate $(0.21 \mathrm{~g}, 1.1$ mmol) and anhydrous $\mathrm{Na}_{2} \mathrm{CO}_{3}(0.74 \mathrm{~g}, 7 \mathrm{mmol})$ were stirred in anhydrous $\mathrm{CH}_{3} \mathrm{CN}(20 \mathrm{~mL})$ at reflux for 20 hours under inert atmosphere. After removal of the solvent, water was added and the product was extracted with $\mathrm{CH}_{2} \mathrm{Cl}_{2}$. The organic phase was washed with water and dried over $\mathrm{MgSO}_{4}$. A chromatography on silica gel (cyclohexane/ $\mathrm{CH}_{2} \mathrm{Cl}_{2}, 70: 30$ ) gave the pure compound $(0.185 \mathrm{~g}, 27 \%) .[\alpha]^{20}{ }_{\mathrm{D}}-6.2^{\circ}\left(1 \mathrm{~g} \cdot \mathrm{L}^{-1}, \mathrm{CH}_{2} \mathrm{Cl}_{2}\right) .{ }^{1} \mathrm{H} \mathrm{NMR}\left(200.1 \mathrm{MHz}, \mathrm{CDCl}_{3}\right) \delta$ 0.91-0.97 (m, 9H, $\left.\mathrm{CH}_{3}\right), 1.15-1.45\left(\mathrm{~m}, 6 \mathrm{H}, \mathrm{CH}_{2}\right), 1.50-1.75\left(\mathrm{~m}, 27 \mathrm{H}, \mathrm{CH}_{3}+\mathrm{CH}_{2}\right), 1.90-2.10$ $\left(\mathrm{m}, 6 \mathrm{H}, \mathrm{OCH}_{2}\right), 3.89\left(\mathrm{~s}, 3 \mathrm{H}, \mathrm{CH}_{3}\right), 4.04\left(\mathrm{br} \mathrm{t}^{3} \mathrm{~J}=6.3 \mathrm{~Hz}, 6 \mathrm{H}, \mathrm{OCH}_{2}\right), 5.06-5.12(\mathrm{~m}, 3 \mathrm{H}, \mathrm{CH})$, 7.26 (s, 2H, H arom.). ${ }^{13} \mathrm{C}\left\{{ }^{1} \mathrm{H}\right\} \mathrm{NMR}\left(75.47 \mathrm{MHz}, \mathrm{CDCl}_{3}\right) 17.6$ (x2), 19.4, 19.5, 25.5, 25.7, $26.8,29.4,29.5,29.7,30.2,36.3,37.2$ (x2), 37.3, 39.9, 43.5, 52.1, 67.4, 71.6, 107.9, 114.2, $124.3,124.7$ (x2), 124.9, 125.1, 129.7, 130.0, 130.9, 131.2, 131.5, 135.2, 142.3, 145.9, 152.3, 152.8, 166.9; UV-vis $\left(\mathrm{CH}_{2} \mathrm{Cl}_{2}, 23{ }^{\circ} \mathrm{C}\right): \lambda \max \left(\varepsilon, \mathrm{M}^{-1} \mathrm{~cm}^{-1}\right)=269$ (10400). IR (KBr) : 3426, 2960, 2919, 2873, 1721 (vCOO), 1676 (vC=C), 1588, 1500, 1433, 1379, 1335, 1216, 1116, 1052, 1016, $914 \mathrm{~cm}^{-1} . \mathrm{MS}(\mathrm{FAB}+, m \mathrm{NBA}): m / z(\%)=599.2(100)[\mathrm{M}+\mathrm{H}]^{+}, 539.2(20)[\mathrm{M}-$ COOMe $]^{+}$Anal. Calcd for $\mathrm{C}_{38} \mathrm{H}_{62} \mathrm{O}_{5}$ : C, 76.21; H, 10.43; Found C, 75.98; H, 10.27.

\section{Methyl 3,4,5-tri(3,7-(S)-dimethyl-6-octyloxy) benzoate (3a)}

To a solution of Methyl 3,4,5-(3,7-(S)-dimethyl-6-octenyloxy)benzoate 2 (0.395 g, $0.7 \mathrm{mmol})$ in $\mathrm{CH}_{2} \mathrm{Cl}_{2} / \mathrm{EtOH}(50: 50,40 \mathrm{~mL})$ was added $1 \mathrm{~mL}$ of water and $\mathrm{Pd} / \mathrm{C}(50 \mathrm{mg})$, the mixture was then stirred under pressure of dihydrogen (40 bars) for 24 hours. After filtration and removal of the solvents, a chromatography on silica gel (cyclohexane/ $\mathrm{CH}_{2} \mathrm{Cl}_{2}$, gradient from 100:0 to 
40:60) gave the attempted compound $(0.36 \mathrm{~g}, 89 \%) .[\alpha]^{20}{ }_{\mathrm{D}}-3.9^{\circ}\left(1 \mathrm{~g} \cdot \mathrm{L}^{-1}, \mathrm{CH}_{2} \mathrm{Cl}_{2}\right) .{ }^{1} \mathrm{H}$ NMR $\left(200.1 \mathrm{MHz}, \mathrm{CDCl}_{3}\right) \delta$ 0.85-0.96 (m, 27H, $\left.\mathrm{CH}_{3}\right), 1.18-1.40\left(\mathrm{~m}, 18 \mathrm{H}, \mathrm{CH}_{2}\right), 1.46-2.02(\mathrm{~m}, 12$

$\left.\mathrm{H}, \mathrm{CH}+\mathrm{CH}_{2}\right), 3.89\left(\mathrm{~s}, 3 \mathrm{H}, \mathrm{CH}_{3}\right), 4.05\left(\mathrm{brt},{ }^{3} \mathrm{~J}=6.2 \mathrm{~Hz}, 6 \mathrm{H}, \mathrm{OCH}_{2}\right), 7.26(\mathrm{~s}, 2 \mathrm{H}) .{ }^{13} \mathrm{C}\left\{{ }^{1} \mathrm{H}\right\}$ DEPT NMR (75.47 MHz, $\left.\mathrm{CDCl}_{3}\right) 19.5\left(\mathrm{CH}_{3}\right), 19.6\left(\mathrm{CH}_{3}\right), 22.6\left(\mathrm{CH}_{3}\right), 22.7\left(\mathrm{CH}_{3}\right), 24.7\left(\mathrm{CH}_{2}\right)$, 24.8 $\left(\mathrm{CH}_{2}\right), 26.9\left(\mathrm{CH}_{2}\right), 27.7\left(\mathrm{CH}_{2}\right), 27.9\left(\mathrm{CH}_{3}\right), 29.6(\mathrm{CH}), 29.8\left(\mathrm{CH}_{3}\right), 30.2\left(\mathrm{CH}_{2}\right), 36.3\left(\mathrm{CH}_{2}\right)$, 37.3 $\left(\mathrm{CH}_{2}\right), 37.5\left(\mathrm{CH}_{2}\right), 39.3\left(\mathrm{CH}_{2}\right), 39.4\left(\mathrm{CH}_{2}\right), 52.1\left(\mathrm{CH}_{3}\right), 67.5\left(\mathrm{CH}_{2}\right), 71.7\left(\mathrm{CH}_{2}\right), 107.9(\mathrm{CH})$, 124.7, 142.4, 152.8, $166.9(\mathrm{C}=\mathrm{O}) \mathrm{UV}$-vis $\left(\mathrm{CH}_{2} \mathrm{Cl}_{2}, 23^{\circ} \mathrm{C}\right): \lambda \max \left(\varepsilon, \mathrm{M}^{-1} \mathrm{~cm}^{-1}\right)=268(15600)$. IR (KBr) : 3430, 2966, 2927, 2870, 1723 (vCOO), 1588, 1500, 1464, 1435, 13834, 1366, $1335,1215,1115,1045,1017,915 \mathrm{~cm}^{-1}$. MS (FAB+, $\left.m \mathrm{NBA}\right): m / z(\%)=605.2(90)[\mathrm{M}+\mathrm{H}]^{+}$, 545.2 (30) [M-COOMe] ${ }^{+}$. Anal. Calcd for $\mathrm{C}_{38} \mathrm{H}_{68} \mathrm{O}_{5}$ : C, 75.45; H, 11.33; Found C, 75.28; H, 11.17.

\section{3,4,5-tri(3,7-(S)-dimethyl-6-octyloxy) benzoic acid (3b)}

A solution of Methyl 3,4,5-tri(3,7-(S)-dimethyl-6-octyloxy) benzoate 3a (0.26 g, $0.4 \mathrm{mmol})$ in EtOH $(15 \mathrm{~mL})$, water $(1 \mathrm{~mL})$ and $\mathrm{KOH}(0.10 \mathrm{~g}, 1.7 \mathrm{mmol})$ was stirred at reflux for 12 hours. After cooling, concentrated $\mathrm{HCl}(10 \%)$ was added to the solution to reach $\mathrm{pH}=2$. The solution was concentrated to half volume and the product was extracted with $\mathrm{CHCl}_{3}$. The organic phase was washed with water and dried over $\mathrm{MgSO}_{4}$. Removal of the solvent furnished a pure colourless oil.(0.23 g, $90 \%)[\alpha]^{20}{ }_{\mathrm{D}}-4.7^{\circ}\left(1 \mathrm{~g} \cdot \mathrm{L}^{-1}, \mathrm{CH}_{2} \mathrm{Cl}_{2}\right) .{ }^{1} \mathrm{H}$ NMR $(300.1$ $\left.\mathrm{MHz}, \mathrm{CDCl}_{3}\right) \delta$ 0.86-0.97 (m, 27H, $\left.\mathrm{CH}_{3}\right), 1.10-1.45\left(\mathrm{~m}, 18 \mathrm{H}, \mathrm{CH}_{2}\right), 1.45-2.1(\mathrm{~m}, 12 \mathrm{H}$, $\left.\mathrm{CH}+\mathrm{CH}_{2}\right), 4.05-4.10\left(\mathrm{~m}, 6 \mathrm{H}, \mathrm{OCH}_{2}\right), 7.36$ (s, 2H, H arom.). ${ }^{13} \mathrm{C}\left\{{ }^{1} \mathrm{H}\right\}$ DEPT NMR (75.47 $\left.\mathrm{MHz}, \mathrm{CDCl}_{3}\right) 19.6(\mathrm{x} 2), 22.6,22.7,24.7\left(\mathrm{CH}_{2}\right), 24.8\left(\mathrm{CH}_{2}\right), 26.9,27.9,29.6,29.7,29.8,30.2$, 36.3, 37.3, 37.5, 39.3( $\left(\mathrm{CH}_{2}\right), 39.4\left(\mathrm{CH}_{2}\right), 43.5\left(\mathrm{CH}_{2}\right), 67.5\left(\mathrm{CH}_{2}\right), 71.7\left(\mathrm{CH}_{2}\right), 108.6(\mathrm{CH}), 123.7$, 143.2, 152.8, $172.2(\mathrm{C}=\mathrm{O})$. UV-vis $\left(\mathrm{CH}_{2} \mathrm{Cl}_{2}, 23{ }^{\circ} \mathrm{C}\right): \lambda \max \left(\varepsilon, \mathrm{M}^{-1} \mathrm{~cm}^{-1}\right)=271(14570) . \mathrm{IR}$ (KBr) : 3381, 2954, 2921, 2870, 1688 (vCOO), 1587, 1502, 1464, 1432, 1383, 1366, 1328, 
$1269,1229,1116,1045,994,955 \mathrm{~cm}^{-1}$. MS (FAB+, $\left.m \mathrm{NBA}\right): m / z(\%)=591.2(60)[\mathrm{M}+\mathrm{H}]^{+}$. Anal. Calcd for $\mathrm{C}_{37} \mathrm{H}_{66} \mathrm{O}_{5}$ : C, 75.20; H, 11.26; Found C, 75.02; H, 11.00.

\section{3,4,5-tri(3,7-(S)-dimethyl-6-octyloxy) benzoyl chloride (3c)}

3,4,5-(3,7-(S)-dimethyl-6-octyloxy) benzoic acid 3b (2.78 g, $4.7 \mathrm{mmol})$ was refluxed in pure $\mathrm{SOCl}_{2}(50 \mathrm{~mL})$ for 3 hours. After removal of the excess of $\mathrm{SOCl}_{2}$, the compound was directly engaged in the next synthesis to avoid degradation.

\section{2,6-bis(3,4,5- trioctyloxybenzoylamino)-4-iodo-toluene (4a)}

To a solution of 2,6 diamino-4-iodo-toluene $1(0.2 \mathrm{~g}, 0.8 \mathrm{mmol})$ in $150 \mathrm{~mL}$ of dry acetone was added 3,4,5-trioctyloxybenzoyl chloride (1.05 g, 2 mmol) and anhydrous $\mathrm{Na}_{2} \mathrm{CO}_{3}(0.43 \mathrm{~g}, 4$ mmol). The mixture was stirred under reflux for 20 hours. The solution was concentrated to half-volume and a white precipitate was obtained after cooling to $-20{ }^{\circ} \mathrm{C}$. The powder was filtered and washed with water in order to remove the unreacted $\mathrm{Na}_{2} \mathrm{CO}_{3}$. The pure product was obtained by successive crystallisation in hot acetone and $\mathrm{CH}_{2} \mathrm{Cl}_{2} / \mathrm{CH}_{3} \mathrm{CN}$ (1:1) mixture $(0.27 \mathrm{~g}, 27 \%) .{ }^{1} \mathrm{H}$ NMR $\left(200.1 \mathrm{MHz}, \mathrm{CDCl}_{3}\right) \delta 0.87\left(\mathrm{~m}, 18 \mathrm{H}, \mathrm{CH}_{3}\right), 1.28-1.33(\mathrm{~m}, 48 \mathrm{H}$, $\left.\mathrm{CH}_{2}\right), 1.43-1.55\left(\mathrm{~m}, 12 \mathrm{H}, \mathrm{CH}_{2}\right), 1.76-1.83\left(\mathrm{~m}, 12 \mathrm{H}, \mathrm{CH}_{2}\right), 2.15\left(\mathrm{~s}, 3 \mathrm{H}, \mathrm{CH}_{3}\right), 4.02(\mathrm{~m}, 12 \mathrm{H}$,

$\left.\mathrm{OCH}_{2}\right), 7.09(\mathrm{~s}, 4 \mathrm{H}), 7.76(\mathrm{~s}, 2 \mathrm{H}), 7.87(\mathrm{~s}, 2 \mathrm{H}) .{ }^{13} \mathrm{C}\left\{{ }^{1} \mathrm{H}\right\}$ DEPT NMR $\left(75.47 \mathrm{MHz}, \mathrm{CDCl}_{3}\right)$ 13.0 $\left(\mathrm{CH}_{3}\right), 14.1\left(\mathrm{CH}_{3}\right), 22.6\left(\mathrm{CH}_{2}\right), 22.7\left(\mathrm{CH}_{2}\right), 26.09\left(\mathrm{CH}_{2}\right), 29.3\left(\mathrm{CH}_{2}\right), 29.4\left(\mathrm{CH}_{2}\right), 29.5\left(\mathrm{CH}_{2}\right)$, 30.3( $\left(\mathrm{CH}_{2}\right), \quad 31.8\left(\mathrm{CH}_{2}\right), 31.9\left(\mathrm{CH}_{2}\right), \quad 69.5\left(\mathrm{OCH}_{2}\right), 73.6\left(\mathrm{OCH}_{2}\right), \quad 90.0(\mathrm{Cq}), \quad 106.0(\mathrm{CH})$, 125.5(Cq), 128.9(Cq), $130.8(\mathrm{CH}), 137.3(\mathrm{Cq}), 141.9(\mathrm{Cq}), 153.3(\mathrm{Cq}).), 165.8(\mathrm{C}=\mathrm{O}) . \mathrm{UV}$-vis $\left(\mathrm{CH}_{2} \mathrm{Cl}_{2}, 23{ }^{\circ} \mathrm{C}\right): \lambda \max \left(\varepsilon, \mathrm{M}^{-1} \mathrm{~cm}^{-1}\right)=275(50440) . \mathrm{IR}(\mathrm{KBr}): 3419(\mathrm{vNH}), 3238(\mathrm{vNH})$, 2922, 2848, $1635(\mathrm{C}=\mathrm{O}), 1581,1510(\delta \mathrm{NH}), 1486,1453,1424,1387,1338,1285,1226$, 1117, $1037 \mathrm{~cm}^{-1}$. MS (FAB+, $\left.m \mathrm{NBA}\right): m / z(\%)=1225.3(100)[\mathrm{M}+\mathrm{H}]^{+}$. Anal. Calcd for $\mathrm{C}_{69} \mathrm{H}_{113} \mathrm{IN}_{2} \mathrm{O}_{8}:$ C, 67.62; H, 9.29 ; N, 2.29; Found C, 67.44; H, 9.07; N, 2.00. 


\section{2,6-bis(3,4,5-tridodecyloxybenzoylamino)-4-iodo-toluene (4b)}

A solution of 2,6-diamino-4-iodo-toluene 1 (0.33 g, $1.3 \mathrm{mmol})$, 3,4,5-tridodecyloxybenzoyl chloride $(2.3 \mathrm{~g}, 3.25 \mathrm{mmol})$ and anhydrous $\mathrm{Na}_{2} \mathrm{CO}_{3}(0.7 \mathrm{~g}, 6.5 \mathrm{mmol})$ in dry acetone $(150 \mathrm{~mL})$ was stirred under reflux for 20 hours. A white precipitate was obtained after cooling to room temperature. The powder was filtered and washed with water in order to remove the unreacted $\mathrm{Na}_{2} \mathrm{CO}_{3}$. The pure product was obtained by successive crystallisation in hot acetone and in a $\mathrm{CH}_{2} \mathrm{Cl}_{2} / \mathrm{CH}_{3} \mathrm{CN}$ mixture (1.8 g, $\left.86 \%\right) .{ }^{1} \mathrm{H}$ NMR (200.1 MHz, $\left.\mathrm{CDCl}_{3}\right) \delta$ 0.85-0.89 (m, 18H, $\mathrm{CH}_{3}$ ), 1.26 (brs, $\left.96 \mathrm{H}, \mathrm{CH}_{2}\right), 1.44-1.56\left(\mathrm{~m}, 12 \mathrm{H}, \mathrm{CH}_{2}\right), 1.76-1.84\left(\mathrm{~m}, 12 \mathrm{H}, \mathrm{CH}_{2}\right), 2.15$ (s, 3H, $\left.\mathrm{CH}_{3}\right), 4.02\left(\mathrm{~m}, 12 \mathrm{H}, \mathrm{OCH}_{2}\right), 7.08(\mathrm{~s}, 4 \mathrm{H}), 7.69(\mathrm{~s}, 2 \mathrm{H}), 7.90(\mathrm{~s}, 2 \mathrm{H}) .{ }^{13} \mathrm{C}\left\{{ }^{1} \mathrm{H}\right\}$ DEPT NMR (75.47 MHz, $\left.\mathrm{CDCl}_{3}\right)$ 13.1( $\left(\mathrm{CH}_{3}\right), 14.1\left(\mathrm{CH}_{3}\right), 22.7\left(\mathrm{CH}_{2}\right), 26.1\left(\mathrm{CH}_{2}\right), 29.3\left(\mathrm{CH}_{2}\right), 29.4\left(\mathrm{CH}_{2}\right)$, $29.6\left(\mathrm{CH}_{2}\right), 29.7\left(\mathrm{CH}_{2}\right), 29.8\left(\mathrm{CH}_{2}\right), 30.3\left(\mathrm{CH}_{2}\right), 31.9\left(\mathrm{CH}_{2}\right), 69.5\left(\mathrm{OCH}_{2}\right), 73.6\left(\mathrm{OCH}_{2}\right)$, 90.0(Cq.), 106.0(CH), 125.4(Cq.), 128.9(Cq.), 130.7 (CH), 137.3(Cq.), 141.9(Cq.), 153.3 (Cq.), $165.7(\mathrm{C}=\mathrm{O})$. UV-vis $\left(\mathrm{CH}_{2} \mathrm{Cl}_{2}, 23{ }^{\circ} \mathrm{C}\right): \lambda_{\max }\left(\varepsilon, \mathrm{M}^{-1} \mathrm{~cm}^{-1}\right)=275$ (30836). IR ( $\left.\mathrm{KBr}\right)$ :

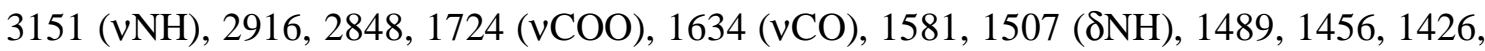
$1387,1337,1282,1223,1145,1112,1072,1029,990 \mathrm{~cm}^{-1} . \mathrm{MS}(\mathrm{FAB}+, m \mathrm{NBA}): m / z(\%)=$ $1562.2(100)[\mathrm{M}+\mathrm{H}]^{+}$. Anal. Calcd for $\mathrm{C}_{93} \mathrm{H}_{161} \mathrm{IN}_{2} \mathrm{O}_{8}: \mathrm{C}, 71.50 ; \mathrm{H}, 10.39$; N, 1.79; Found $\mathrm{C}$, 71.29; H, 10.17; N, 1.59.

\section{2,6-bis(3,4,5-trihexadecyloxybenzoylamino)-4-iodo-toluene (4c)}

A mixture of 2,6-diamino-4-iodo-toluene 1 (0.19 g, $0.8 \mathrm{mmol})$, 3,4,5-trihexadecyloxybenzoyl chloride $(1.72 \mathrm{~g}, 2 \mathrm{mmol})$ and anhydrous $\mathrm{Na}_{2} \mathrm{CO}_{3}(0.43 \mathrm{~g}, 4 \mathrm{mmol})$ in dry acetone $(150 \mathrm{~mL})$ was stirred under reflux for 15 hours. A white powder precipitated after cooling to room temperature and was removed by filtration and washed with water. The pure product was obtained by successive crystallisation in hot acetone and in a $\mathrm{CH}_{2} \mathrm{Cl}_{2} / \mathrm{CH}_{3} \mathrm{CN}$ mixture $(1.1 \mathrm{~g}$, 
$76 \%$ ). ${ }^{1} \mathrm{H}$ NMR $\left(200.1 \mathrm{MHz}, \mathrm{CDCl}_{3}\right) \delta 0.85-0.89\left(\mathrm{~m}, 18 \mathrm{H}, \mathrm{CH}_{3}\right), 1.26\left(\right.$ br s, $\left.144 \mathrm{H}, \mathrm{CH}_{2}\right)$, 1.45-1.53 (m, 12H, $\left.\mathrm{CH}_{2}\right) 1.76-1.84\left(\mathrm{~m}, 12 \mathrm{H}, \mathrm{CH}_{2}\right), 2.16\left(\mathrm{~s}, 3 \mathrm{H}, \mathrm{CH}_{3}\right), 4.03\left(\mathrm{~m}, 12 \mathrm{H}, \mathrm{OCH}_{2}\right)$, $7.08(\mathrm{~s}, 4 \mathrm{H}), 7.70(\mathrm{~s}, 2 \mathrm{H}), 7.90(\mathrm{~s}, 2 \mathrm{H}) .{ }^{13} \mathrm{C}\left\{{ }^{1} \mathrm{H}\right\} \operatorname{NMR}\left(75.47 \mathrm{MHz}, \mathrm{CDCl}_{3}\right)$ 13.1, 14.1, 22.7, $26.1,29.4,29.5,29.6,29.7,30.3,30.9,31.9,69.5,73.6,90.0,106.0,128.9,130.7,137.3$, 141.9, 153.3, 165.7. UV-vis $\left(\mathrm{CH}_{2} \mathrm{Cl}_{2}, 23{ }^{\circ} \mathrm{C}\right): \lambda \max \left(\varepsilon, \mathrm{M}^{-1} \mathrm{~cm}^{-1}\right)=275$ (53660). IR $(\mathrm{KBr})$ : $3457(v \mathrm{NH}), 3181(\mathrm{vNH}), 2910,2849,1635(\mathrm{vCO}), 1580,1507(\delta \mathrm{NH}), 1489,1470,1425$, 1387, 1338, 1288, 1227, 1118, $1013987 \mathrm{~cm}^{-1}$. MS (FAB+, $\left.m \mathrm{NBA}\right): \mathrm{m} / z(\%)=1898.2(100)$ $[\mathrm{M}+\mathrm{H}]^{+}$. Anal. Calcd for $\mathrm{C}_{117} \mathrm{H}_{209} \mathrm{IN}_{2} \mathrm{O}_{8}: \mathrm{C}, 74.01 ; \mathrm{H}, 11.09 ; \mathrm{N}, 1.48$; Found C, 73.81; H, $10.75 ; \mathrm{N}, 1.15$.

\section{2,6-bis[3,4,5-tri(3,7-(S)-dimethyl-6-octyloxy)-benzoylamino]-4-iodo-toluene (4d)}

3,4,5-(3,7-(S)-dimethyl-6-octyloxy) benzoyl chloride 3c (2.86 g, $4.7 \mathrm{mmol}), 2,6$ diamino-4iodo-toluene $1(0.465 \mathrm{~g}, 1.9 \mathrm{mmol})$ and $\mathrm{Na}_{2} \mathrm{CO}_{3}(1.00 \mathrm{~g}, 9.4 \mathrm{mmol})$ were mixed in dry acetone $(150 \mathrm{~mL})$. The solution was heated at reflux under inert atmosphere for 20 hours. The $\mathrm{Na}_{2} \mathrm{CO}_{3}$ excess was filtered out hot and diamine was precipitate in acetone solution at low temperature $\left(-20{ }^{\circ} \mathrm{C}\right)$. A chromatography on silica gel (cyclohexane $/ \mathrm{CH}_{2} \mathrm{Cl}_{2}$, gradient from 50:50 to 30:70) gave the desired compound $(0.86 \mathrm{~g}, 33 \%) \cdot[\alpha]^{20}{ }_{\mathrm{D}}-4.0^{\circ}\left(1 \mathrm{~g} \cdot \mathrm{L}^{-1}, \mathrm{CH}_{2} \mathrm{Cl}_{2}\right) .{ }^{1} \mathrm{H}$ $\operatorname{NMR}\left(200.1 \mathrm{MHz}, \mathrm{CDCl}_{3}\right) \delta 0.87\left(\mathrm{~m}, 54 \mathrm{H}, \mathrm{CH}_{3}\right), 1.04-1.41\left(\mathrm{~m}, 36 \mathrm{H}, \mathrm{CH}_{2}\right), 1.41-1.99(\mathrm{~m}, 24$ $\left.\mathrm{H}, \mathrm{CH}+\mathrm{CH}_{2}\right), 2.17\left(\mathrm{~s}, 3 \mathrm{H}, \mathrm{CH}_{3}\right), 4.04\left(\mathrm{~m}, 12 \mathrm{H}, \mathrm{OCH}_{2}\right), 7.08$ (s, $4 \mathrm{H}, \mathrm{H}$ arom.), 7.67 (s, $2 \mathrm{H}, \mathrm{H}$ arom.), 7.91 (s, 2H, NH). ${ }^{13} \mathrm{C}\left\{{ }^{1} \mathrm{H}\right\}$ DEPT NMR (75.47 MHz, $\left.\mathrm{CDCl}_{3}\right) 12.7\left(\mathrm{CH}_{3}\right), 19.4,19.5$, 22.6, 22.7, $24.7\left(\mathrm{x} 2\left(\mathrm{CH}_{2}\right)\right), 26.9\left(\mathrm{CH}_{2}\right), 28.0,29.8,30.2\left(\mathrm{CH}_{2}\right), 36.4\left(\mathrm{CH}_{2}\right), 37.4\left(\mathrm{CH}_{2}\right)$, 37.5 $\left(\mathrm{CH}_{2}\right), 37.6\left(\mathrm{CH}_{2}\right), 39.3\left(\mathrm{CH}_{2}\right), 39.4\left(\mathrm{CH}_{2}\right), 43.5,67.6\left(\mathrm{CH}_{2}\right), 71.7\left(\mathrm{CH}_{2}\right), 89.5,106.1(\mathrm{CH})$, 126.5, 128.6, $131.6(\mathrm{CH}), 136.9,141.8,153.3,166.1(\mathrm{C}=\mathrm{O})$. UV-vis $\left(\mathrm{CH}_{2} \mathrm{Cl}_{2}, 23{ }^{\circ} \mathrm{C}\right): \lambda \max$ $\left(\varepsilon, \mathrm{M}^{-1} \mathrm{~cm}^{-1}\right)=275(33200) . \mathrm{IR}(\mathrm{KBr}): 3265(\mathrm{vNH}), 3107(\mathrm{vNH}), 2924,1635(v \mathrm{CO}), 1582$, $1554,1515(\delta \mathrm{NH}), 1470,1453,1335,1113,1044,998,970,918 \mathrm{~cm}^{-1} . \mathrm{MS}(\mathrm{FAB}+, m \mathrm{NBA})$ : 
$m / z(\%)=1394.2(95)[\mathrm{M}+\mathrm{H}]^{+}, 1393.2(100)[\mathrm{M}]^{+}, 1265.3(20)[\mathrm{M}-\mathrm{I}]^{+}$Anal. Calcd for $\mathrm{C}_{81} \mathrm{H}_{137} \mathrm{IN}_{2} \mathrm{O}_{8}:$ C, 69.80; H, 9.91; N, 2.01; Found C, 69.69; H, 9.84; N, 1.72.

\section{2,6-bis(3,4,5-tridodecyloxybenzoylamino)-4-trimethylsilylethynyl-toluene (5a)}

A schlenk flask was charged with 2,6-bis(3,4,5-tridodecyloxybenzoylamino)-4-iodo-toluene 4b $(0.506 \mathrm{~g}, 0.324 \mathrm{mmol}), \mathrm{Pd}\left(\mathrm{PPh}_{3}\right)_{2} \mathrm{Cl}_{2}(0.014 \mathrm{~g}, 0.019 \mathrm{mmol}), \mathrm{THF}(50 \mathrm{~mL})$ and $(i \mathrm{Pr})_{2} \mathrm{NH}$ (5 mL). The mixture was exhaustively degassed with argon. Trimethylsilylacetylene $(0.069$ $\mathrm{mL}, 0.486 \mathrm{mmol})$ and $\mathrm{CuI}(0.013 \mathrm{~g}, 0.06 \mathrm{mmol})$ were then added and the mixture was stirred at room temperature overnight. The organic phase was filtered over celite, washed with water (150 mL) and dried over $\mathrm{MgSO}_{4}$. After removal of the solvent, a chromatography on silica gel (cyclohexane/ $\mathrm{CH}_{2} \mathrm{Cl}_{2}$, gradient from 50:50 to 0:100) afforded the pure titled compound (0.636 g, 73\%). ${ }^{1} \mathrm{H}$ NMR (200.1 MHz, $\left.\mathrm{CDCl}_{3}\right) \delta 0.13\left(\mathrm{~s}, 9 \mathrm{H}, \mathrm{Si}-\mathrm{CH}_{3}\right)$ 0.85-0.89 (m, 18H, $\left.\mathrm{CH}_{3}\right), 1.28$ (br s, $96 \mathrm{H}, \mathrm{CH}_{2}$ ), 1.43-1.57 (m, 12H, $\left.\mathrm{CH}_{2}\right), 1.80-1.85\left(\mathrm{~m}, 12 \mathrm{H}, \mathrm{CH}_{2}\right), 2.20\left(\mathrm{~s}, 3 \mathrm{H}, \mathrm{CH}_{3}\right), 4.00$ (m, 12H, OCH 2$), 7.09$ (s, 4H, H arom.), 7.61 (s, 2H, H arom.), 7.72 (s, 2H, NH). ${ }^{13} \mathrm{C}\left\{{ }^{1} \mathrm{H}\right\}$ DEPT NMR (75.47 MHz, $\left.\mathrm{CDCl}_{3}\right)-0.2\left(\mathrm{Si}_{-} \mathrm{CH}_{3}\right), 13.4\left(\mathrm{CH}_{3}\right), 14.1\left(\mathrm{CH}_{3}\right), 22.7\left(\mathrm{CH}_{2}\right)$, 26.1 $\left(\mathrm{CH}_{2}\right), 29.3\left(\mathrm{CH}_{2}\right), 29.4\left(\mathrm{CH}_{2}\right), 29.6\left(\mathrm{CH}_{2}\right), 29.7\left(\mathrm{x} 2\left(\mathrm{CH}_{2}\right)\right), 29.8\left(\mathrm{CH}_{2}\right), 30.4\left(\mathrm{CH}_{2}\right), 31.9$ $\left(\mathrm{CH}_{2}\right), 69.5\left(\mathrm{OCH}_{2}\right), 73.6\left(\mathrm{OCH}_{2}\right), 94.7(\mathrm{Cq}),. 104.1(\mathrm{Cq}),. 106.0(\mathrm{CH}), 121.5(\mathrm{Cq}),. 125.7(\mathrm{CH}$ arom.), 127.3(Cq.), 129.1(Cq.), 136.3(Cq.), 141.8(Cq.), 153.3 (Cq.), 165.8 (C=O). UV-vis $\left(\mathrm{CH}_{2} \mathrm{Cl}_{2}, 23{ }^{\circ} \mathrm{C}\right): \lambda \max \left(\varepsilon, \mathrm{M}^{-1} \mathrm{~cm}^{-1}\right)=254$ (25560), 266 (34900), 279 (19020). IR (KBr) : $3304(\mathrm{vNH}), 3190(\mathrm{vNH}), 2915,2850,2152,1635$ (vCO), 1582, $1504(\delta \mathrm{NH}), 1491,1470$, $1427,1390,1338,1261,1235,1114,1018 \mathrm{~cm}^{-1} . \mathrm{MS}(\mathrm{FAB}+, m \mathrm{NBA}): \mathrm{m} / z(\%)=1532.2(100)$ $[\mathrm{M}+\mathrm{H}]^{+}, 1458.2(25)\left[\mathrm{M}-\mathrm{Si}\left(\mathrm{CH}_{3}\right)_{3}\right]^{+}$. Anal. Calcd for $\mathrm{C}_{98} \mathrm{H}_{170} \mathrm{~N}_{2} \mathrm{O}_{8} \mathrm{Si}: \mathrm{C}, 76.81 ; \mathrm{H}, 11.18$; N, 1.83; Found C, 76.50; H, 11.04; N, 1.63. 


\section{2,6-bis[3,4,5-tri(3,7-(S)-dimethyl-6-octyloxy)-benzoylamino]-4-trimethylsilylethynyl-}

\section{toluene $(5 \mathbf{b})$}

A schlenk flask was charged with 2,6-bis(3,4,5-(3,7-(S)-dimethyl-6-octyloxy)benzoylamino)-4-iodo-toluene $4 \mathbf{d}(0.489 \mathrm{~g}, 0.350 \mathrm{mmol}), \mathrm{Pd}\left(\mathrm{PPh}_{3}\right)_{2} \mathrm{Cl}_{2}(0.015 \mathrm{~g}, 0.021$ mmol), distilled THF $(50 \mathrm{~mL})$ and $\left({ }^{\mathrm{i}} \mathrm{Pr}\right)_{2} \mathrm{NH}(5 \mathrm{~mL})$. The mixture was thoroughly degassed with argon. trimethylsilylacetylene $(0.08 \mathrm{~mL}, 0.525 \mathrm{mmol})$ and $\mathrm{CuI}(0.007 \mathrm{~g}, 0.04 \mathrm{mmol})$ were then added and the mixture was stirred at room temperature overnight. The organic phase was filtered over celite, washed with water $(150 \mathrm{~mL})$ and dried over $\mathrm{MgSO}_{4}$. After removal of the solvent, a chromatography on silica gel (cyclohexane $/ \mathrm{CH}_{2} \mathrm{Cl}_{2}, 50: 50$ ) afforded the titled compound $(0.395 \mathrm{~g}, 83 \%)$. $[\alpha]^{20}{ }_{\mathrm{D}}-5.0^{\circ}\left(1 \mathrm{~g} \cdot \mathrm{L}^{-1}, \mathrm{CH}_{2} \mathrm{Cl}_{2}\right) .{ }^{1} \mathrm{H}$ NMR $(300.1 \mathrm{MHz}$, $\left.\mathrm{CDCl}_{3}\right) \delta 0.16\left(\mathrm{~s}, 9 \mathrm{H}, \mathrm{CH}_{3}\right), 0.84-1.02(\mathrm{~m}, 54 \mathrm{H}), 1.04-1.41(\mathrm{~m}, 36 \mathrm{H}), 1.41-1.99(\mathrm{~m}, 24 \mathrm{H})$, $2.18(\mathrm{~s}, 3 \mathrm{H}), 4.03-4.06\left(\mathrm{~m}, 12 \mathrm{H}, \mathrm{OCH}_{2}\right), 7.13(\mathrm{~s}, 4 \mathrm{H}), 7.54(\mathrm{~s}, 2 \mathrm{H}), 7.87(\mathrm{~s}, 2 \mathrm{H}) .{ }^{13} \mathrm{C}\left\{{ }^{1} \mathrm{H}\right\}$ DEPT NMR (75.47 MHz, $\left.\mathrm{CDCl}_{3}\right)-0.16,13.4,19.6,22.6$ (x2), 22.7, 24.7( $\left.\mathrm{CH}_{2}\right)$, 28.0, 29.7, 29.8, 36.4 ( $\left(\mathrm{CH}_{2}\right), 37.4\left(\mathrm{CH}_{2}\right), 37.5\left(\mathrm{CH}_{2}\right), 39.3\left(\mathrm{CH}_{2}\right), 39.4\left(\mathrm{CH}_{2}\right), 67.7\left(\mathrm{OCH}_{2}\right), 71.8\left(\mathrm{OCH}_{2}\right)$, 94.8(Cq), 104.1(Cq), $105.9(\mathrm{CH}), 121.5(\mathrm{Cq}), 125.7(\mathrm{CH}), 127.4(\mathrm{Cq}), 129.1(\mathrm{Cq}), 136.3(\mathrm{Cq})$, 141.7(Cq), $153.3(\mathrm{Cq}), 165.9(\mathrm{C}=\mathrm{O})$. UV-vis $\left(\mathrm{CH}_{2} \mathrm{Cl}_{2}, 23{ }^{\circ} \mathrm{C}\right): \lambda \max \left(\varepsilon, \mathrm{M}^{-1} \mathrm{~cm}^{-1}\right)=253$ (48990), 266 (59060), 277 (38920). IR (KBr) : 3205 (vNH), 2952, 2919, 2851, 2160, 1638 (vCO), 1607, 1582, 1518 ( $\delta \mathrm{NH}), 1459,1426,1383,1336,1292,1234,1113,1045,997,942$ $\mathrm{cm}^{-1} . \mathrm{MS}(\mathrm{FAB}+, m \mathrm{NBA}): m / z(\%)=1364.2(100)[\mathrm{M}+\mathrm{H}]^{+}, 1265.2(10)\left[\mathrm{M}-\mathrm{CCSiMe}_{3}\right]^{+}$ Anal. Calcd for $\mathrm{C}_{86} \mathrm{H}_{146} \mathrm{~N}_{2} \mathrm{O}_{8}$ Si: C, 75.72; H, 10.79; N, 2.05; Found C, 75.52; H, 10.67; N, 1.83 .

\section{2,6-bis(3,4,5-tridodecyloxybenzoylamino)-4-ethynyl-toluene (6a)}

A solution of 2,6-bis(3,4,5-tridodecyloxybenzoylamino)-4-trimethylsilylethynyl-toluene 5a (0.32 g, $0.2 \mathrm{mmol})$ and potassium fluoride $(0.18 \mathrm{~g}, 4 \mathrm{mmol})$ in $\mathrm{CH}_{2} \mathrm{Cl}_{2} / \mathrm{MeOH}(80: 20,50 \mathrm{~mL})$ 
was stirred at room temperature for 24 hours. The organic phase was washed with water (150 $\mathrm{mL}$ ) and dried over $\mathrm{MgSO}_{4}$. After removal of the solvent, a chromatography on silica gel with $\left(\mathrm{CH}_{2} \mathrm{Cl}_{2} / \mathrm{MeOH}, 99.9: 0.1\right)$ gave the desired compound as a white powder $(0.19 \mathrm{~g}, 62 \%) .{ }^{1} \mathrm{H}$ NMR (300.1 MHz, $\left.\mathrm{CDCl}_{3}\right) \delta$ 0.86-0.89 (m, 18H, $\left.\mathrm{CH}_{3}\right), 1.28\left(\right.$ br s, $\left.96 \mathrm{H}, \mathrm{CH}_{2}\right), 1.43-1.55(\mathrm{~m}$, $\left.12 \mathrm{H}, \mathrm{CH}_{2}\right), 1.78-1.83\left(\mathrm{~m}, 12 \mathrm{H}, \mathrm{CH}_{2}\right), 2.19\left(\mathrm{~s}, 3 \mathrm{H}, \mathrm{CH}_{3}\right), 4.03\left(\mathrm{~m}, 12 \mathrm{H}, \mathrm{OCH}_{2}\right), 7.10(\mathrm{~s}, 4 \mathrm{H})$, $7.61(\mathrm{~s}, 2 \mathrm{H}), 7.78(\mathrm{~s}, 2 \mathrm{H}) .{ }^{13} \mathrm{C}\left\{{ }^{1} \mathrm{H}\right\}$ DEPT NMR (75.47 MHz, $\left.\mathrm{CDCl}_{3}\right)$ 13.3( $\left.\mathrm{CH}_{3}\right), 14.1\left(\mathrm{CH}_{3}\right)$, 22.7 $\left(\mathrm{CH}_{2}\right), \quad 26.1\left(\mathrm{CH}_{2}\right), \quad 29.3\left(\mathrm{CH}_{2}\right), \quad 29.4\left(\mathrm{CH}_{2}\right), \quad 29.6\left(\mathrm{CH}_{2}\right), 29.7\left(\mathrm{x} 2\left(\mathrm{CH}_{2}\right)\right), \quad 29.8\left(\mathrm{CH}_{2}\right)$, 30.4 $\left(\mathrm{CH}_{2}\right), 31.9\left(\mathrm{CH}_{2}\right), 55.8,69.5\left(\mathrm{OCH}_{2}\right), 73.6\left(\mathrm{OCH}_{2}\right), 82.7,106.0(\mathrm{CH}), 120.6,125.7(\mathrm{CH})$, 129.0, 136.4, 141.8, 153.3, $165.9(\mathrm{C}=\mathrm{O})$. UV-vis $\left(\mathrm{CH}_{2} \mathrm{Cl}_{2}, 23{ }^{\circ} \mathrm{C}\right): \lambda \max \left(\varepsilon, \mathrm{M}^{-1} \mathrm{~cm}^{-1}\right)=254$ (35510), 274 (34780). IR (KBr) : 3422 (vNH), 2915, 2848, 1634 (vCO), 1579, 1507 ( $\delta \mathrm{NH})$, $1489,1438,1423,1384,1338,1221,1113,983 \mathrm{~cm}^{-1} . \mathrm{MS}(\mathrm{FAB}+, m \mathrm{NBA}): \mathrm{m} / z(\%)=1460.2$ (100) $[\mathrm{M}+\mathrm{H}]^{+}$. Anal. Calcd for $\mathrm{C}_{95} \mathrm{H}_{162} \mathrm{~N}_{2} \mathrm{O}_{8}: \mathrm{C}, 78.14 ; \mathrm{H}, 11.18 ; \mathrm{N}, 1.92$; Found $\mathrm{C}, 77.70 ; \mathrm{H}$, $10.80 ; \mathrm{N}, 1.57$.

\section{2,6-bis[3,4,5-tri(3,7-(S)-dimethyl-6-octyloxy)-benzoylamino]-4-ethynyl-toluene (6b).}

To a solution of 2,6-bis[3,4,5-tri(3,7-(S)-dimethyl-6-octyloxy)-benzoylamino]-4trimethylsilylethynyl-toluene $\mathbf{5 b}(0.25 \mathrm{~g}, 0.18 \mathrm{mmol})$ in THF $(100 \mathrm{~mL})$ was added $2 \mathrm{~mL}$ of $\mathrm{NaOH}$ methanol solution $\left(\mathrm{C}=1 \mathrm{~mol} \cdot \mathrm{L}^{-1}\right)$. The solution was stirred for 1 hour at room temperature. The solution was neutralized with saturated $\mathrm{NH}_{4} \mathrm{Cl}$ solution $(\sim 50 \mathrm{~mL})$. The

product was extracted with $\mathrm{CH}_{2} \mathrm{Cl}_{2}$ and dried over $\mathrm{MgSO}_{4}$. Recrystallization in a $\mathrm{CH}_{2} \mathrm{Cl}_{2} / \mathrm{CH}_{3} \mathrm{CN}$ mixture gave the pure desired compound $(0.19 \mathrm{~g}, 80 \%)$. $[\alpha]^{20}{ }_{\mathrm{D}}-3.8^{\circ}\left(1 \mathrm{~g} . \mathrm{L}^{-1}\right.$, $\mathrm{CH}_{2} \mathrm{Cl}_{2}$ ). ${ }^{1} \mathrm{H}$ NMR $\left(400.1 \mathrm{MHz}, \mathrm{CDCl}_{3}\right) \delta$ 0.84-1.02 (m, $\left.54 \mathrm{H}\right), 1.02-1.41(\mathrm{~m}, 36 \mathrm{H}), 1.41-$ $1.99(\mathrm{~m}, 24 \mathrm{H}), 2.11(\mathrm{~s}, 3 \mathrm{H}), 2.97(\mathrm{~s}, 1 \mathrm{H}), 3.95-4.15\left(\mathrm{~m}, 12 \mathrm{H}, \mathrm{OCH}_{2}\right), 7.18(\mathrm{~s}, 4 \mathrm{H}), 7.40$ (s, 2H), $8.24(\mathrm{~s}, 2 \mathrm{H}) .{ }^{13} \mathrm{C}\left\{{ }^{1} \mathrm{H}\right\}$ DEPT NMR (75.47 MHz, $\left.\mathrm{CDCl}_{3}\right)$ 13.9, 19.9 (x2), 23.0, 23.1, 25.1 $\left(\mathrm{CH}_{2}\right), 28.4,30.1,30.2,36.8\left(\mathrm{CH}_{2}\right), 37.8\left(\mathrm{CH}_{2}\right), 37.9\left(\mathrm{CH}_{2}\right), 39.7\left(\mathrm{CH}_{2}\right), 39.8\left(\mathrm{CH}_{2}\right)$, 
68.1( $\left(\mathrm{CH}_{2}\right), 72.2\left(\mathrm{CH}_{2}\right), 83.1,106.3(\mathrm{CH}), 120.7(\mathrm{Cq}), 126.4(\mathrm{CH}), 128.6(\mathrm{Cq}), 129.4(\mathrm{Cq})$, 136.8(Cq), 142.1(Cq), $153.7(\mathrm{Cq}), 166.4(\mathrm{C}=\mathrm{O})$. UV-vis $\left(\mathrm{CH}_{2} \mathrm{Cl}_{2}, 23^{\circ} \mathrm{C}\right): \lambda \max \left(\varepsilon, \mathrm{M}^{-1} \mathrm{~cm}^{-1}\right)$ $=253$ (43400), 275 (43100). IR (KBr) : $3183(\mathrm{vNH}), 2947,2919,2863,1634(\mathrm{vCO}), 1581$, $1515(\delta \mathrm{NH}), 1486,1456,1424,1384,1337,1234,1115,1044,994 \mathrm{~cm}^{-1} . \mathrm{MS}(\mathrm{FAB}+$, $m$ NBA): $m / z(\%)=1291.2(100)[\mathrm{M}]^{+}$. Anal. Calcd for $\mathrm{C}_{83} \mathrm{H}_{138} \mathrm{~N}_{2} \mathrm{O}_{8}: \mathrm{C}, 77.16 ; \mathrm{H}, 10.77 ; \mathrm{N}$, 2.17; Found C, 77.01; H, 10.59; N, 1.84 .

\section{5-[2,6-bis(3,4,5-tridodecyloxybenzoylamino)-4-ethynyl-toluene]-2,2'-bipyridine (7).}

A Schlenk flask was charged with 2,6-bis(3,4,5-tridodecyloxybenzoylamino)-4-ethynyltoluene 6a $(0.400 \mathrm{~g}, 0.320 \mathrm{mmol}), \mathrm{Pd}\left(\mathrm{PPh}_{3}\right)_{2} \mathrm{Cl}_{2}(0.018 \mathrm{~g}, 0.032 \mathrm{mmol})$, THF $(20 \mathrm{~mL})$ and $(i \mathrm{Pr})_{2} \mathrm{NH}(3 \mathrm{~mL})$. The mixture was exhaustively degassed with argon. Then, 5-ethynyl-2,2'bipyridine $(0.046 \mathrm{mg}, 0.320 \mathrm{mmol})$ and $\mathrm{CuI}(0.006 \mathrm{~g}, 0.0384 \mathrm{mmol})$ were then added and the mixture was stirred at room temperature overnight. The organic phase was filtered over celite, washed with water $(150 \mathrm{~mL})$ and dried over $\mathrm{MgSO}_{4}$. After removal of the solvent, a chromatography on silica gel pre-treated with triethylamine using (hexane $/ \mathrm{CH}_{2} \mathrm{Cl}_{2}$, gradient from 70:30 to 50:50) afforded the pure titled compound 7 (0.325 g, 63\%). ${ }^{1} \mathrm{H}$ NMR (200.1 $\left.\mathrm{MHz}, \mathrm{CDCl}_{3}\right) \delta 8.78(\mathrm{~s}, 1 \mathrm{H}), 8.71\left(\mathrm{dd}, 1 \mathrm{H},{ }^{3} \mathrm{~J}=7.9 \mathrm{~Hz},{ }^{4} \mathrm{~J}=1.0 \mathrm{~Hz}\right), 7.92(\mathrm{~m}, 2 \mathrm{H}), 7.85(\mathrm{~s}$, 1H), $7.75(\mathrm{~s}, 2 \mathrm{H}), 7.36\left(\mathrm{dd}, 2 \mathrm{H},{ }^{3} J=7.9 \mathrm{~Hz},{ }^{4} \mathrm{~J}=1.0 \mathrm{~Hz}\right), 7.29(\mathrm{~s}, 2 \mathrm{H}), 7.16(\mathrm{~s}, 4 \mathrm{H}), 4.07$ (m, $12 \mathrm{H}), 2.27(\mathrm{~s}, 3 \mathrm{H}), 1.82(\mathrm{~m}, 12 \mathrm{H}), 1.52(\mathrm{~m}, 12 \mathrm{H}), 1.31(\mathrm{~m}, 90 \mathrm{H}), 0.92(\mathrm{~m}, 18 \mathrm{H}) .{ }^{13} \mathrm{C}\left\{{ }^{1} \mathrm{H}\right\}$

DEPT NMR (75.47 MHz, $\left.\mathrm{CDCl}_{3}\right) \quad 13.7\left(\mathrm{CH}_{3}\right), \quad 14.5\left(\mathrm{CH}_{3}\right), \quad 23.1\left(\mathrm{CH}_{2}\right), \quad 26.5\left(\mathrm{CH}_{2}\right)$, 29.8 $\left(2 \mathrm{xCH}_{2}\right), \quad 29.9\left(\mathrm{CH}_{2}\right), \quad 30.0\left(\mathrm{CH}_{2}\right), \quad 30.1\left(\mathrm{CH}_{2}\right), \quad 30.2\left(2 \mathrm{xCH}_{2}\right), \quad 30.8\left(\mathrm{CH}_{2}\right), 32.3\left(\mathrm{CH}_{2}\right)$, 32.4 $\left(\mathrm{CH}_{2}\right), \quad 69.9\left(\mathrm{OCH}_{2}\right), \quad 74.0\left(\mathrm{OCH}_{2}\right), \quad 93.1(\mathrm{C} \equiv \mathrm{C}), \quad 87.2(\mathrm{C} \equiv \mathrm{C}), \quad 106.4(\mathrm{CH}), \quad 120.5(\mathrm{Cq})$, 120.7(CH), $121.5(\mathrm{Cq}), 121.7(\mathrm{CH}), 124.3(\mathrm{CH}), 125.6(\mathrm{CH}), 127.5(\mathrm{Cq}), 129.5(\mathrm{Cq}), 137.0$ $(\mathrm{Cq}), 137.3(\mathrm{CH}), 139.7(\mathrm{CH}), 142.3(\mathrm{Cq}), 149.7(\mathrm{CH}), 152.3(\mathrm{CH}), 153.7(\mathrm{Cq}), 155.3(\mathrm{Cq})$, $155.9(\mathrm{Cq}), 166.3(\mathrm{Cq}) . \mathrm{UV}$-vis $\left(\mathrm{CH}_{2} \mathrm{Cl}_{2}, 23{ }^{\circ} \mathrm{C}\right): \lambda \max \left(\varepsilon, \mathrm{M}^{-1} \mathrm{~cm}^{-1}\right)=287(41650), 320$ 
(37200), 339 (30500), 366 (4900). IR (KBr) : 3233 (vNH), 2910, 2848, 2851, 1635 (vCO), $1583,1526(\delta \mathrm{NH}), 1492,1463,1422,1386,1335,1236,1119,1043,1022,986 \mathrm{~cm}^{-1} . \mathrm{MS}$ $(\mathrm{FAB}+, m \mathrm{NBA}): m / z(\%)=1614.2(100)[\mathrm{M}+\mathrm{H}]^{+}, 1444.2(20)\left[\mathrm{M}^{-} \mathrm{C}_{12} \mathrm{H}_{25}\right]^{+}$. Anal. Calcd for $\mathrm{C}_{105} \mathrm{H}_{168} \mathrm{~N}_{4} \mathrm{O}_{8}: \mathrm{C}, 78.11 ; \mathrm{H}, 10.49 ; \mathrm{N}, 3.47$; Found C, 77.84; H, 10.27; N, 3.31.

\section{Bis-5,5'-[2,6-bis(3,4,5-tridodecyloxybenzoylamino)-4-ethynyl-toluene]-2,2'-bipyridine (8)}

A Schlenk flask was charged with 2,6-bis(3,4,5-tridodecyloxybenzoylamino)-4-ethynyltoluene 6a $(0.255 \mathrm{~g}, 0.163 \mathrm{mmol}), \mathrm{Pd}\left(\mathrm{PPh}_{3}\right)_{2} \mathrm{Cl}_{2}(0.006 \mathrm{~g}, 0.016 \mathrm{mmol})$, THF $(25 \mathrm{~mL})$ and $(i \mathrm{Pr}){ }_{2} \mathrm{NH}(5 \mathrm{~mL})$. The mixture was exhaustively degassed with argon. Then, 5,5'-diethynyl2,2'-bipyridine $(0.017 \mathrm{mg}, 0.081 \mathrm{mmol})$ and $\mathrm{CuI}(0.002 \mathrm{~g}, 0.006 \mathrm{mmol})$ were then added and the mixture was stirred at room temperature overnight. The organic phase was filtered over celite, washed with water $(150 \mathrm{~mL})$ and dried over $\mathrm{MgSO}_{4}$. After removal of the solvent, a chromatography on silica gel pre-treated with triethylamine using $\mathrm{CH}_{2} \mathrm{Cl}_{2}$, as solvent affording the pure titled compound $(0.163 \mathrm{~g}, 65 \%) .{ }^{1} \mathrm{H}$ NMR $\left(200.1 \mathrm{MHz}, \mathrm{CDCl}_{3}\right) \delta 8.62(\mathrm{~s}$, 2H), $8.22\left(\mathrm{~d}, 1 \mathrm{H},{ }^{3} J=7.9 \mathrm{~Hz}\right), 8.09(\mathrm{~s}, 1 \mathrm{H}), 7.74\left(\mathrm{~d}, 1 \mathrm{H},{ }^{3} J=7.9 \mathrm{~Hz}\right), 7.60(\mathrm{~s}, 2 \mathrm{H}), 7.10$ (s, $4 \mathrm{H}), 3.99(\mathrm{~m}, 12 \mathrm{H}), 2.21(\mathrm{~s}, 3 \mathrm{H}), 1.75(\mathrm{~m}, 12 \mathrm{H}), 1.26(\mathrm{~m}, 102 \mathrm{H}), 0.87(\mathrm{~m}, 18 \mathrm{H}) .{ }^{13} \mathrm{C}\left\{{ }^{1} \mathrm{H}\right\}$ DEPT NMR (75.47 MHz, $\left.\mathrm{CDCl}_{3}\right) \quad 13.9\left(\mathrm{CH}_{3}\right), \quad 14.6\left(\mathrm{CH}_{3}\right), \quad 23.4\left(\mathrm{CH}_{2}\right), \quad 26.3\left(\mathrm{CH}_{2}\right)$, 29.7 $\left(2 \mathrm{xCH}_{2}\right), \quad 30.1\left(2 \mathrm{xCH}_{2}\right), \quad 30.5\left(\mathrm{CH}_{2}\right), 30.6\left(\mathrm{CH}_{2}\right), 30.4\left(2 \mathrm{xCH}_{2}\right), \quad 30.8\left(\mathrm{CH}_{2}\right), 32.5\left(\mathrm{CH}_{2}\right)$, 32.8( $\left(\mathrm{CH}_{2}\right), 70.0\left(\mathrm{OCH}_{2}\right), 74.3\left(\mathrm{OCH}_{2}\right), 93.4(\mathrm{C} \equiv \mathrm{C}), 87.5(\mathrm{C} \equiv \mathrm{C}), 106.6(\mathrm{CH}), 120.5(\mathrm{Cq}), 121.5$ $(\mathrm{Cq}), 121.5(\mathrm{CH}), 125.4(\mathrm{CH}), 127.5(\mathrm{Cq}), 129.5(\mathrm{Cq}), 137.0(\mathrm{Cq}), 139.9(\mathrm{CH}), 142.3(\mathrm{Cq})$, 152.1(CH), $153.7(\mathrm{Cq}), 155.9(\mathrm{Cq}), 166.3(\mathrm{C}=\mathrm{O}) . \mathrm{UV}$-vis $\left(\mathrm{CH}_{2} \mathrm{Cl}_{2}, 23{ }^{\circ} \mathrm{C}\right): \lambda \max \left(\varepsilon, \mathrm{M}^{-1} \mathrm{~cm}^{-}\right.$ $\left.{ }^{1}\right)=277$ (97800), 352 (69600), 370 (55000). IR (KBr) : $3178(\mathrm{vNH}), 2914,2851,2213,1634$ (vCO), 1581, $1521(\delta \mathrm{NH}), 1486,1467,1424,1388,1337,1266,1228,1115,1025,994,951$ $\mathrm{cm}^{-1}$. MS $(\mathrm{FAB}+, m \mathrm{NBA}): m / z(\%)=3071.2(100)[\mathrm{M}+\mathrm{H}]^{+}$. Anal. Calcd for $\mathrm{C}_{200} \mathrm{H}_{328} \mathrm{~N}_{6} \mathrm{O}_{16}$ : C, 78.17; H, 10.76; N, 2.73; Found C, 77.84; H, 10.43; N, 2.62. 